\title{
A CAGE EFFECT IN COLLISION-INDUCED LIGHT ABSORPTION FROM A MOLECULAR DYNAMICS CALCULATION
}

\author{
J. Courtenay LEWIS and J.A. TJON \\ Institute for Theoretical Physics, University of Utrecht, Urecht, The Netherlands \\ Received 19 September 1974
}

\begin{abstract}
A computer experiment on a two-dimensional Lorentz gas is deveribed for which the autocorrelation function of the induced dipcle moment exhibits an extremely long positive tail. It is argued that this tail car be ascribed to the trapping of particles in irregular cages.
\end{abstract}

The collision-induced unfrared spectra of homonuclear diatomic molecules, and the collision-induced far infrared translational spectra of inert gas mixtures; arise from the transitory electric dipole moments by the intermolecular interaction of colliding molecules. These spectra characteristically show interference effects due to an underlying time correlation in the intermolecular force $[1,2]$. Such intercollisional interference effects manifest themselves in the induced dipole moment autocorrelation function as a long negative tail. In a well-known molecular dynamics calculation [3], Alder and Wainwright found that the velocity autocorrelation function for a fluid of hard disks showed interesting structure at large times; which they explained as due to a persistent hydrodynamic vortex flow, though it has subsequently been obtained from low density expansions $[4,5]$. In contrast, virtually no attempts have been made to examine the autocorrelation functions for internal degrees of freedom for long times and at high densities. In this note we report a molecular dynamics simulation which shows clear evidence for an additional positive tail in the incuced dipole moment autocorrelation function at high densities which is longer even than the negative interierence contribution. This long positive tail apparently is due to the trapping of particles in asymmetric cages of unlike particles.

$\therefore$ The model which ve have studied is a two-dimensional Lorentz gas similar to that used recently in a numerical experiment on the validity of low density expansions for the diffusion coefficient [6]. It con- sists of one light particle of radius $\sigma$ which moves in a box with periodic boundary conditions and undergoes collisions with $N$ randomly distributed hard disks of radius $\sigma_{0}$ fixed at positions $r_{i}$. We assume a pairwise additive induced dipole moment of the form

$\mu(r)=\sum_{i=1}^{N} \frac{r-r_{i}}{\left|r-r_{i}\right|} \mu_{0}\left(\left|r-r_{i}\right|\right)$

where $r$ is the position of the light particle, and

$\mu_{0}(R)=m_{0} \exp (-R / \rho)$.

We generate the configurations of the fixed scatterers using a staridard Monte Carlo technique [7]. The light particle is placed at random in such a configuration subjec! to the condition that it does not overlap a fixed scatterer and the direction of its velocity is chosen at random. Its trajectory is then followed for a time $T$. The induced dipole moment $\mu_{l}(r(t))$ is calculated from the trajectory, and the time autocorrelation function

$C_{l}(\tau)=\frac{1}{T-\tau} \int_{0}^{T-\tau} \mathrm{d} t \mu_{l}(r(t+\tau)) \cdot \mu_{l}(r(t))$

is formed. This process is repeated for $N_{c}$ different startinj configurations, after which the configuration averaged induced dipole moment autocorrelation function

$C(\tau)=\frac{1}{N_{\mathrm{c}}} \sum_{l=1}^{N_{\mathrm{c}}} C_{l}(\tau)$

is calculated. We take $\sigma+\sigma_{0}=1$ and the speed of the light particle to be 1. For the sake of clarity the resul- 


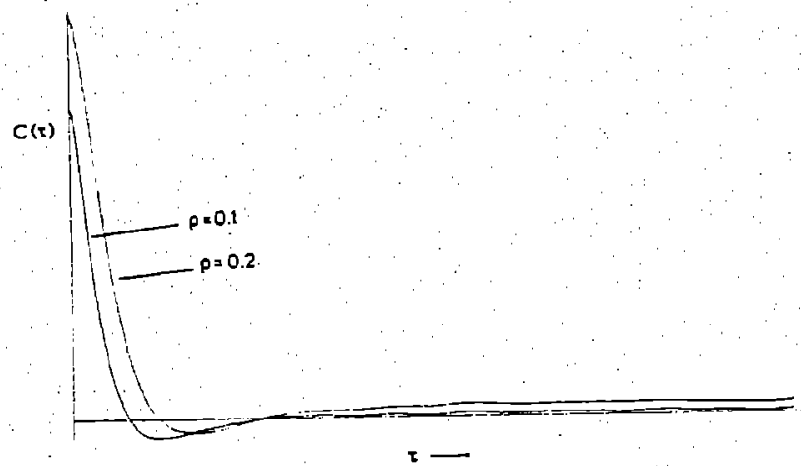

Fig.1. The autocorrelation function $C(\tau)$ as a function of $\tau$ for two values of the range parameter $\rho$.

tant spectra are not velocity averaged; the velocity average is in any case of a trivial nature for this system. Unless otherwise indicated the ratio $\sigma / \sigma_{0}$ chosen was that for $\mathrm{H}_{2}-\mathrm{Ar}$ [8]. In most of the computer runs $N$ was taken to be 225 paiticles and the number of configurations $N_{\mathrm{c}}$ of the order of 300 . We took $\rho$ in the range $0.05-0.20 . T$ was 125 time units which at high density was sufficient to allow several hundred to a few thousand collisions, depending on the configuration chosen. The autocorrelation function $C(\tau)$ obtained from the estimate (3) is only useful for $\tau \leqslant$ $0.1 T$ as the variance becomes large for $\tau$ of the order of $T$ [9]. The system was studied at various densities between $A / A_{0}=23$ and $A / A_{0}=1.425$, where $A$ is the area of the bcx and $A_{0}$ the close-packed area. At low densities $C(\tau)$ shows a long negative intercollisional interference tail, as expected. The corresponding power spectrum $W(\omega)$ shows a dip about zero frequency, the dip extending to about $\frac{1}{2}$ of the peak height. $W(\omega)$ was obtained by discrete Fourier transformation of $C(\tau)$. At a density of $A / A_{0}=5.7$ a sinall positive peak appears in the bowl of the intercollisional interference dip in $W(\omega)$. This positive peak becomes more prominent as the density is increased. $C(\tau)$ and $W(\omega)$ are shown in fịs. 1 and 2 , respectively, for the highest density considered. In these two computer runs, $N_{\mathrm{c}}=320$, and the same configurations and trajectories were use $d$ for both values of the range parameter $\rho . C(\tau)$ is shown for $\tau$ between 0 and 6.2 . The positive tail is evident. It in fact shows no sensible diminution from 6.2 to 12.5 , the largest yalue of $\tau$ for which we calculated $C(\tau)$. The width of the central peak in $W(\omega)$ is independent of $p$ and is only four times the frequency step of the discrete Fourier trans-

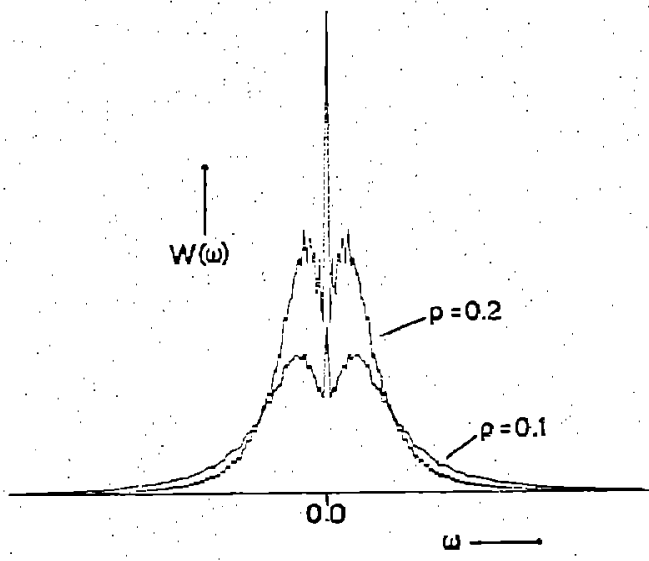

Fig. 2. The power spectrum $W(\omega)$ as a function of the frequency $\omega$ for two values of the range parameter $p$.

form used. Both $C(\tau)$ and $W(\omega)$ show considerable fluctuation. Unfortunately, significant reduction in the fluctuation would require prohibitive amounts of computer time.

The positive tail of the autocorrelation function can be interpreted qualitatively as due to the fact that at such high densities the fixed scatterers tend to form cages in which the light particle will be trapped for long periods of time. During the stay of a light particle in an irregular cage the time average of its induced dipole moment will in general differ from zero, and as can be seen imriediately from eqs. (3) and (4), this leads to an autocorrelation function with a positive tail. The decay time for the tail is the escape time for a particle from one cage to another. For perfectly caged particles, $C(\tau)$ will never decrease to zero.

In order to see whether the above picture is correct we formed the correlation function

$$
\begin{aligned}
& \widetilde{C}(\tau)=\frac{1}{N_{\mathrm{c}}} \sum_{l=1}^{N_{\mathrm{c}}} \frac{1}{T-\tau} \\
& \quad \times \int_{0}^{T-\tau} \mathrm{d} t\left[\mu_{l}(r(\tau+\tau))-\bar{\mu}_{l}\right] \cdot\left[\mu_{l}(r(t))-\overline{\mathrm{p}}_{l} \mathrm{I}\right.
\end{aligned}
$$

for the same configuration and trajectories, where $\bar{\mu}_{i}$ is the time averaged induced dipole moment in configuration $l$,

$\bar{\mu}_{l}=\frac{1}{T} \int_{0}^{T} \mathrm{~d} t \mu_{l}(r(t))$ 
$\widetilde{C}(\tau)$ so computed appsars to remain negative, but goes to zero from below in a faw mean free times. There is no central spike in the corresponding power spectrum. It is worthwhile noting that the time average of $\mu$ over the interval $T$ is not identical to the configurational average of $\mu$ which is consistent with zero for the cases considered here. Heric if $\boldsymbol{\mu}$ is to be eigo. dic; then much longer times than the $T$ which we. have used are necessary to establish the equality of the two averages.

To assess the effects of cage irregularity we made one run in a regular "face-centred squlare". lattice with $\sigma=0$ and with $\mu_{0}(R) \propto 1 / R^{4}$. The central spike was quite absent. In a second run, with the same $\sigma$ and induced dipole moment, at a sightiy lower density, but with a random configuration generated in the usual way, a definite though not pronounced spike was seen.

If the induced dipole moment were proportional to the intermolecular force on the light particle, then $W(\omega)$ would of necessity go to zero at zero frequency, and the spike would be absent $[10,11]$. It is thus to be expected that the greater the range of the induced dipole in our model, the larger the spike. Such tums out to be the case. The spike for $p=0.2$ is clearly more prominent than that for $\rho=0.1$, while those for $\rho=0.05$ and for $\mu_{0} \propto \exp \left[-(R-1)^{2} / 0.1\right]$ (not shown) are less so. The spike for a $1 / R^{4}$ induced dipole moment is larger even than that for $\rho=0.2$.

The above point aiso touches on the possibility of observing such a peak in a physical system. As pointed out in ref. [10], the quantity $R \equiv W(0) / W W_{\max }$ is in a sense a measure of the similarity or dissimilarity of the intermolecular force to the induced dipole moment. By that measure, the in duced dipole moment in $\mathrm{H}_{2}$-rare gas systems $[2,12]$, for which $\leftarrow R \leqslant 0.1$, is much more similar to the intermolecular force than is $\mu$ in our model, for which $R \approx 0.1$. This factor alone would reduce the size of a possible spike, as would the finite lifetime of the cages. Further, the averaging of $\mu$ over the third dimension is more likely to reduce than enhance it. However, it is not impossible that a small positive feature seen in the intercollisional interference dip in the $\mathrm{Q}$ branch of the $\mathrm{p}: \mathrm{H}_{2}-$ As fundamental band [12] might originate in caging. As for the quadrupole-induced dipole moment re- sponsible for the $\mathrm{S}$ branch, while it has a $1 / R^{4}$ dependence such as would give a large caging effect in our model, in a physical system this wili be diminished very much by the angular dependence of $\mu$, and will be smeared out by velocity averaging and by collisional pertirbation of the vibrational rotational transition frequency. Our caging effect would thus be difficult to clistinguish from diffusional narrowing, with which it is in any case conceptually related [13].

This investigation is part of a research program of the "Stishting voor Fundamenteel Orderzoek der Materie" and was made possible by financial support from the "Nederlandse Organisatie voor Zuiver-Wetenschappelijk Onderzoek": We are indebted to the Computation Centre of the Rijksuniversiteit Utrecht for the use of their CDC 6500 . We are pleased to acknowleclge useful and interesting discussions with Dr. M.H. Erast.

\section{References}

[1] I.C. Lewis and J. van Kranendonk, Phys. Rev. Letters 24 (1970) 802; Can. J. Phys 50 (1972) 352.

[2] J.W. Mactaggart and H.L. Welsh, Car. J. Phys 51 (1973) 158.

[3] B.J. Alder and T.E. Wainwright, Phys. Rcv. Letters 18 (19157) 988; Phys. Rev. A1 (1970) 18 .

[4] M.F., Einst, E.H. Hauge and J.M.J. van Leeuwen, Phys. Rev, Letters 25 (1970) 1254.

[5] J.R Dorfman ard E.G.D. Cohen, Phys. Rev. Letters 25 (1970) 1257.

[6] C. E ruin, Phys. Rev. Letters 25 (1972) 1670; Physica 72 (1974) 261.

[7] N. Metropolis; A.W.Rosenbluth, M.N. Rosenbluth, A.M. Teller and E. Teller, J. Chem. Phys. 21 (1953) 1087.

[8] A.R.W. McKeliar and H.L. Welsh, J. Chem. Phys. 55 (1971) 595.

[9] R.B. Blackman and J.W. Tukey, The measurement of power spectra (Dover, New York, 1959); D. Middleton, Statistical communication theory (Mc Graw-Hill, New York, 1960).

[10] J.C. Lewis, Can. J. Phys. 51 (1973) 2455.

[11] J. ven Kranendonk, Can. J. Phys. 46 (1968) 1173.

[12] J.W. Mactaggart, Ph.D. Thesis, University of Toronto, Torunto, Canada (1971), unpublished.

[13] J.ven Kuanendonk and D.M. Gass, Can. J. Phys 51 (1973) 2420 . 